\title{
Alternative separation of Laplace's equation in toroidal coordinates and its application to electrostatics
}

\author{
Mark Andrews \\ Physics, The Faculties, Australian National University, ACT 0200, Australia \\ Received 1 November 2004; received in revised form 25 July 2005; accepted 25 November 2005 \\ Available online 28 December 2005
}

\begin{abstract}
The usual method of separation of variables to find a basis of solutions of Laplace's equation in toroidal coordinates is particularly appropriate for axially symmetric applications; for example, to find the potential outside a charged conducting torus. An alternative procedure is presented here that is more appropriate where the boundary conditions are independent of the spherical coordinate $\theta$ (rather than the toroidal coordinate $\eta$ or the azimuthal coordinate $\psi$ ). Applying these solutions to electrostatics leads to solutions, given as infinite sums over Legendre functions of the second kind, for (i) an arbitrary charge distribution on a circle, (ii) a point charge between two intersecting conducting planes, (iii) a point charge outside a conducting half plane. In the latter case, a closed expression is obtained for the potential. Also the potentials for some configurations involving charges inside a conducting torus are found in terms of Legendre functions. For each solution in the basis found by this separation, reconstructing the potential from the charge distribution (corresponding to singularities in the solutions) gives rise to integral relations involving Legendre functions.
\end{abstract}

(C) 2005 Elsevier B.V. All rights reserved.

Keywords: Laplace equation; Separation of variables; Toroidal coordinates; Legendre polynomials

\section{Introduction}

The method of separation of variables in various coordinate systems is a classic approach to finding exact solutions of Laplace's equation and has been thoroughly studied [1]. One such set of coordinates is the toroidal system, but it will be argued here that some of the usefulness of this coordinate system has been hidden because, while the usual way of separating the variables is appropriate for some situations, there is another way that is more suited to a certain class of problems, in particular some interesting problems in electrostatics.

The toroidal coordinates [2] of any point are given by the intersection of a torus, a sphere with its centre on the axis of the torus (the $z$-axis), and an azimuthal half plane (terminated by the $z$-axis). The radius and centre of the sphere are determined by the spherical coordinate $\theta$, the major and minor radii of the torus are given by the toroidal coordinate $\eta$, and the particular half plane is

E-mail address: mark.andrews@anu.edu.au. specified by its azimuthal angle $\psi$. The scale of the coordinates is determined by a length $a$ (see Fig. 1). The details of this orthogonal coordinate system are reviewed in Section 2.

The traditional method of solving Laplace's equation by separation of these variables [2] gives a complete basis of solutions of the form $(\cosh \eta-\cos \theta)^{1 / 2} f(\eta) \Theta(\theta) \Psi(\psi)$, where $f(\eta)$ is an associated Legendre function $P_{p-1 / 2}^{q}$ $(\cosh \eta)$ or $Q_{p-1 / 2}^{q}(\cosh \eta), \Theta(\theta)$ is $\sin p \theta$ or $\cos p \theta$, and $\Psi(\psi)$ is $\sin q \psi$ or $\cos q \psi$. This basis is particularly convenient for axially symmetric situations, for then we set $q=0$ and the solution involves the Legendre functions $P_{p-1 / 2}(\cosh \eta)$ or $Q_{p-1 / 2}(\cosh \eta)$ (rather than the associated Legendre functions). This type of solution can be found in several textbooks [3-5]. An example from electrostatics is the potential due to a charged conducting torus; this and several other examples are briefly discussed in Appendix B.

Here, we show that there is an alternative separation that gives a basis of the form $r^{-1 / 2} f(\eta) \Theta(\theta) \Psi(\psi)$, where $r=$ $a \sinh \eta /(\cosh \eta-\cos \theta)$ is the distance from the $z$-axis, $f(\eta)$ is $P_{v-1 / 2}^{\mu}(\operatorname{coth} \eta)$ or $Q_{v-1 / 2}^{\mu}(\operatorname{coth} \eta), \Theta(\theta)$ is $\sin \mu \theta$ or $\cos \mu \theta$, 


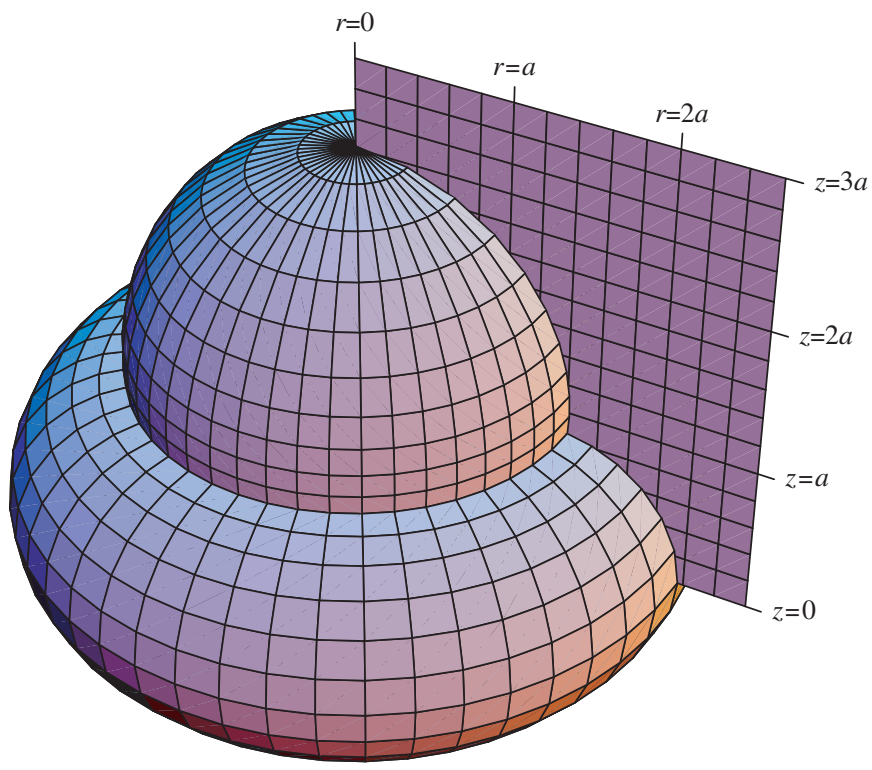

Fig. 1. The toroidal coordinates of any point are given by the intersection of a sphere, a torus, and an azimuthal half plane. The torus shown here has $\eta=1$ and the sphere has sphere $\theta=\pi / 4$. [Then, according to Eq. (1), $r \approx 1.40 a$ and $z \approx 0.84 a$.]

and $\Psi(\psi)$ is $\sin v \psi$ or $\cos v \psi$. This basis is more convenient for situations where the boundary conditions do not involve $\theta$, for then we set $\mu=0$ and the solution involves the Legendre functions $P_{v-1 / 2}(\cosh \eta)$ or $Q_{v-1 / 2}(\cosh \eta)$. We will see that there are some interesting configurations in electrostatics where the boundary conditions are of this type. The potential due to an arbitrary distribution of charge on a circle can be found in this way, and the method can be used even when conducting half planes (terminated by the $z$-axis) are also present. This enable us, for example, to find an expression, as an infinite sum over Legendre functions, for the potential due to a point charge between two intersecting conducting planes. In the case of a point charge outside a single half plane, the infinite series can be summed to give a closed expression for the potential. It is also possible to deal with some distributions of fixed charge inside a portion of a conducting torus when the ends of the portion are closed off by conducting planes. These matters are discussed in Sections 5 and 6.

The singularities of the solutions (of Laplace's equation) found by this separation can be interpreted, in the context of electrostatics, as distributions of charge. Reconstructing the potentials from such a charge distribution (by adding the Coulomb potentials) gives rise to some integrals involving the Legendre functions, including Heine's 1881 representation for $Q_{n-1 / 2}$ and an apparently new integral that can be expressed in terms of $P_{\alpha}$. Approaching these relations from the separation of Laplace's equation throws light on the work of Cohl et al. [6], who were mainly interested in the gravitational applications of the theory.

\section{Toroidal coordinates}

In the toroidal system, the location of a point is given by the coordinates $\eta, \theta, \psi$ where the cartesian coordinates are $(x, y, z)=(a / \Delta)(\sinh \eta \cos \psi, \sinh \eta \sin \psi, \sin \theta)$

with $\Delta:=\cosh \eta-\cos \theta$. (The notation $A:=B$ indicates that $A$ is defined to be $B$.) Thus, $\psi$ is an azimuthal angle denoting a rotation about the $z$-axis, and the distance from this axis is

$r=(a / \Delta) \sinh \eta$.

The range of the coordinates is $\eta \geqslant 0,-\pi<\theta \leqslant \pi, 0 \leqslant \psi<2 \pi$. A little algebra shows that $r^{2}+z^{2}+a^{2}=2 a^{2} \Delta^{-1}$ $\cosh \eta=2 a r \operatorname{coth} \eta$, and the relation

$\operatorname{coth} \eta=\frac{r^{2}+z^{2}+a^{2}}{2 a r}$

will be often used below. Following from this equation, the surfaces of constant $\eta$ are given by

$(r-a \operatorname{coth} \eta)^{2}+z^{2}=a^{2} / \sinh ^{2} \eta$.

For any fixed $\eta$ this is the torus generated by rotating about the $z$-axis a circle $C$ of radius $a / \sinh \eta$ centred at $r=a \operatorname{coth} \eta, z=0$. As $\eta \rightarrow \infty$ this radius becomes small and the torus collapses to the circle $r=a, z=0$, which will be referred to as the reference circle. As $\eta \rightarrow 0$ both the radius, and the distance to the centre, of the circle $C$ become large; then that part of the torus that is within a finite distance of the origin, coincides with the $z$-axis.

In the derivation of Eq. (3), $2 a^{2} \Delta^{-1} \cosh \eta$ can also be written as $2 a^{2}+2 a z \cot \theta$, so the surfaces of constant $\theta$ are given by

$r^{2}+(z-a \cot \theta)^{2}=a^{2} / \sin ^{2} \theta$.

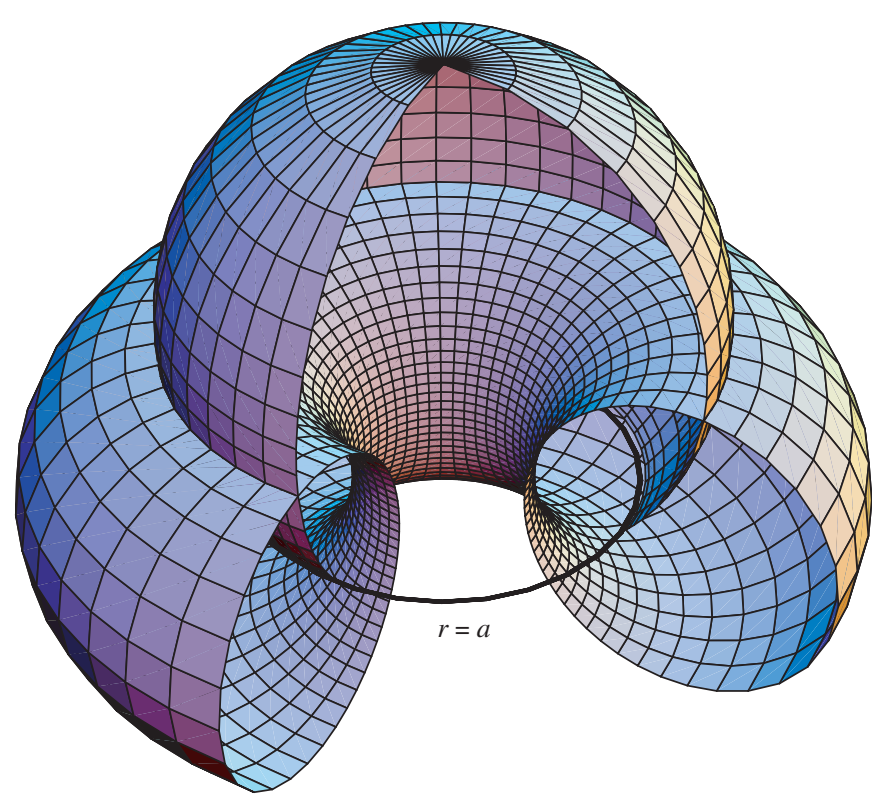

Fig. 2. The reference circle, $r=a, z=0$ of the coordinate system is the intersection of the sphere with the plane $z=0$. For any $\eta$ it lies inside the torus. 
For any fixed $\theta$ this is a sphere of radius $a /|\sin \theta|$, centred on $z=a \cot \theta, r=0$. This sphere intersects the plane $z=0$ in the reference circle. (See Fig. 2).

\section{Alternative separation of Laplace's equation}

In toroidal coordinates Laplace's equation $\nabla^{2} V=0$ becomes [2]

$$
\begin{aligned}
& \partial_{\eta}\left(\Delta^{-1} \sinh \eta \partial_{\eta} V\right)+\partial_{\theta}\left(\Delta^{-1} \sinh \eta \partial_{\theta} V\right) \\
& \quad+(\Delta \sinh \eta)^{-1} \partial_{\psi \psi} V=0,
\end{aligned}
$$

which is not immediately separable. But inserting $V=$ $U / \sqrt{r}$ into this equation gives

$\sinh ^{2} \eta\left(\partial_{\eta \eta} U+\partial_{\theta \theta} U\right)+\partial_{\psi \psi} U+\frac{1}{4} U=0$,

which does separate giving solutions that are products of $f(\eta), \sin \mu \theta$ or $\cos \mu \theta$, and $\sin v \psi$ or $\cos v \psi$, where

$\sinh ^{2} \eta\left(f^{\prime \prime}-\mu^{2} f\right)-\left(v^{2}-\frac{1}{4}\right) f=0$.

Changing variable from $\eta$ to $\chi:=\operatorname{coth} \eta$ converts this equation to

$\left(\chi^{2}-1\right) f^{\prime \prime}+2 \chi f^{\prime}-\left(\frac{\mu^{2}}{\chi^{2}-1}+v^{2}-\frac{1}{4}\right) f=0$,

and the solutions of this are the associated Legendre functions $P_{v-1 / 2}^{\mu}(\chi)$ and $Q_{v-1 / 2}^{\mu}(\chi)$. Therefore, the solutions of Laplace's equation are products of $\sqrt{a / r}, P_{v-1 / 2}^{\mu}(\operatorname{coth} \eta)$ or $Q_{v-1 / 2}^{\mu}(\operatorname{coth} \eta), \sin \mu \theta$ or $\cos \mu \theta$, and $\sin v \psi$ or $\cos v \psi$. This is a complete basis; but we will consider only solutions with $\mu=0$, which still allows arbitrary dependence on $\eta$ and $\psi$.

Table 1

The asymptotic behaviour of the Legendre functions near the singular points $w=1$ and $w=\infty$

\begin{tabular}{lll}
\hline & $P_{\alpha}(w)$ & $Q_{\alpha}(w)$ \\
\hline$w=1$ & $1+\frac{1}{2} \alpha(\alpha+1)(w-1)$ & $-\gamma-\psi(\alpha+1)-\frac{1}{2} \ln \frac{w-1}{2}$ \\
$w=\infty$ & $\frac{\Gamma\left(\alpha+\frac{1}{2}\right)}{\sqrt{\pi} \Gamma(\alpha+1)}(2 w)^{\alpha}$ & $\frac{\sqrt{\pi} \Gamma(\alpha+1)}{\Gamma\left(\alpha+\frac{3}{2}\right)}(2 w)^{-\alpha-1}$ \\
\hline
\end{tabular}

\section{Singularities in the solutions}

From the differential equation (7), singularities of the Legendre functions $P_{\alpha}(w)$ or $Q_{\alpha}(w)$, as a function of the complex variable $w$, can occur only for $w= \pm 1$ or $w=\infty$. We need consider only the region $w \geqslant 1$. Care is needed in accessing information about these functions, because these singularities, if present, are branch points and produce some ambiguities. Formulae or numerical values appropriate for applications that involve $w<1$ may not be valid here. For example, in Mathematica, the function denoted by LegendreQ $(\alpha, w)$ is not satisfactory for our purposes; instead we must use LegendreQ $(\alpha, 0,3, w)$, which is their notation for the Legendre function of the second kind of type 3 . For this function the $w$-plane is not cut for $w \geqslant 1$. For the $\mathrm{P}$ variety of Legendre function, either $\operatorname{LegendreP}(\alpha, w)$ or LegendreP $(\alpha, 0,3, w)$ can be used, because these two functions are identical for $w \geqslant 1$. The asymptotic behaviour [7,8] near $w=1$ and $w=\infty$ is given in Table 1 , except that $P_{-1 / 2}(w) \sim \pi^{-1} \sqrt{2 / w} \ln (8 w)$ as $w \rightarrow \infty$. Here, $\gamma$ is Eulers constant and $\psi(\alpha)$ is the digamma function $\Gamma^{\prime}(\alpha) / \Gamma(\alpha)$.

Now apply the asymptotic behaviour in Table 1 to find the behaviour of $\sqrt{a / r} P_{v-1 / 2}(\chi)$ and $\sqrt{a / r} Q_{v-1 / 2}(\chi)$ as functions of $r$ and $z$. Singularities can occur only for $r=0$ or for $\chi=1$ or $\chi \rightarrow \infty$. From $\chi=\left(r^{2}+z^{2}+a^{2}\right) /(2 a r)$ it follows that $\chi \geqslant 1$, and $\chi=1$ occurs only on the reference circle. Also $\chi \rightarrow \infty$ either for $r \rightarrow 0$ or for $R \rightarrow \infty$, where $R:=\sqrt{r^{2}+z^{2}}$ is the distance from the origin. The asymptotic behaviour in these three regions is given in Table 2, where $C_{v}:=\pi^{-1 / 2} \Gamma(v) / \Gamma\left(v+\frac{1}{2}\right) \quad$ and $\quad D_{v}:=\pi^{1 / 2} \Gamma\left(v+\frac{1}{2}\right) /$ $\Gamma(v+1)$, and where $d:=\left[(r-a)^{2}+z^{2}\right]^{1 / 2}$ is the distance from the reference circle.

Thus, the $Q$-solutions are bounded as $r \rightarrow 0$, have logarithmic singularities at the reference circle, and decrease as $1 / R$ or faster as $R \rightarrow \infty$. They therefore correspond in electrostatics to some finite distribution of charge on the reference circle. The $P$-solutions diverge too rapidly, both for $r \rightarrow 0$ and for $R \rightarrow \infty$, to correspond to finite distributions of charge. They are not singular on the reference circle. They will be shown in Section 7 to be the potentials due to distributions of charge along the $z$-axis; but these distributions are not integrable, so the total charge along the $z$-axis is infinite.

Table 2

The asymptotic behaviour of $\sqrt{a / r} P_{v-1 / 2}(\chi)$ and $\sqrt{a / r} Q_{v-1 / 2}(\chi)$ in the three regions (i) $r \ll a$ (near the $z$-axis), (ii) $d \ll a$ (near the reference circle),

\begin{tabular}{|c|c|c|c|c|}
\hline & $\chi$ & $\sqrt{a / r} P_{v-1 / 2}(\chi)(v>0)$ & $\sqrt{a / r} P_{-1 / 2}(\chi)$ & $\sqrt{a / r} Q_{v-1 / 2}(\chi)$ \\
\hline$r \ll a$ & $\frac{z^{2}+a^{2}}{2 a r}$ & $C_{v}\left(\frac{z^{2}+a^{2}}{a^{2}}\right)^{v-1 / 2}\left(\frac{a}{r}\right)^{v}$ & $\frac{2}{\pi} \frac{a}{\sqrt{z^{2}+a^{2}}} \ln \frac{4\left(z^{2}+a^{2}\right)}{a r}$ & $D_{v}\left(\frac{z^{2}+a^{2}}{a^{2}}\right)$ \\
\hline$d \ll a$ & $1+\frac{d^{2}}{2 a^{2}}$ & 1 & 1 & $-\gamma-\psi\left(v+\frac{1}{2}\right)$ \\
\hline$R \gg a$ & $\frac{R^{2}}{2 a r}$ & $C_{v}\left(\frac{R^{2}}{a^{2}}\right)^{v-1 / 2}\left(\frac{a}{r}\right)^{v}$ & $\frac{2}{\pi} \frac{a}{R} \ln \frac{4 R^{2}}{a r}$ & $D_{v}\left(\frac{R^{2}}{a^{2}}\right)$ \\
\hline
\end{tabular}
and (iii) $R \gg a$ (far from the origin) 


\section{Charge distributed around a ring}

To consider the potential due to a ring of charge, we use toroidal coordinates with the ring as reference circle. Continuity of the potential at $\psi=2 \pi$ requires that $v$ be an integer. The simplest case is where $v=0$ so that the potential has no dependence on the azimuthal angle $\psi$. Then

$V_{0}:=\sqrt{a / r} Q_{-1 / 2}(\chi)$

satisfies Laplace's equation except on the reference circle and, from Table 2 , approaches $\pi a / R$ as $R \rightarrow \infty$. It is therefore the potential due to a charge of $q=4 \pi^{2} \varepsilon_{0} a$ distributed uniformly around the reference circle. Near this circle, from Table 2, $V_{0} \sim \ln (8 a / d)$. Therefore, applying Gauss's law, the line-charge density on the reference circle is $2 \pi \varepsilon_{0}$, which gives the same total charge $q$.

When $v=n$, an integer,

$$
V_{n}:=\sqrt{a / r} Q_{n-1 / 2}(\chi) \cos n \psi
$$

can be analysed in a similar way, and of course $\sin n \psi$ would do just as well as $\cos n \psi$. Near the reference circle $\sqrt{a / r} Q_{n-1 / 2}(\chi)$ has the same limit as for $n=0$; so the linecharge density corresponding to $V_{n}$ is $2 \pi \varepsilon_{0} \cos n \psi$. The total charge is zero; so as $R \rightarrow \infty, V_{n}$ falls off faster than $1 / R$. In the case of $n=1$, one half of the ring has positive charge while the other half is negative. Then the ring will have a dipole moment that can be deduced to be $2 \sqrt{\pi} \varepsilon_{0} a^{2}$ from the asymptotic behaviour $V_{1} \sim \frac{1}{2} \sqrt{\pi} a^{2} R^{-3} r \cos \psi$.

Since we have found the potential for any sinusoidal linecharge density on the reference circle, we can find the potential due to any distribution of charge on the reference circle by expressing it as a Fourier series. The only case that will be dealt with explicitly here is the delta function; this will give the potential due to a point charge. The appropriate Fourier series, for functions that match both in magnitude and derivative at $\psi=0$ and $\psi=2 \pi$, is [9]

$$
\begin{aligned}
\delta\left(\psi-\psi^{\prime}\right) & =\frac{1}{2 \pi} \sum_{n=-\infty}^{\infty} \mathrm{e}^{i n\left(\psi-\psi^{\prime}\right)} \\
& =\frac{1}{2 \pi} \sum_{n=0}^{\infty} \delta_{n} \cos n\left(\psi-\psi^{\prime}\right),
\end{aligned}
$$

where $\delta_{0}=1$ and $\delta_{n}=2$ for $n=1,2,3, \ldots$. Since a linecharge density of $2 \pi \varepsilon_{0} \cos n \phi$ produced the potential $\sqrt{a / r} Q_{n-1 / 2}(\chi) \cos n \phi$, it follows that a point charge $q$ at angle $\psi^{\prime}$ on the reference circle, which will have line-charge density $(q / a) \delta\left(\psi-\psi^{\prime}\right)$, will produce the potential

$$
\begin{aligned}
V_{\delta} & =\frac{q}{4 \pi^{2} \varepsilon_{0} \sqrt{a r}} \sum_{n=0}^{\infty} \delta_{n} Q_{n-1 / 2}(\chi) \cos n\left(\psi-\psi^{\prime}\right) \\
& =\frac{q}{4 \pi^{2} \varepsilon_{0} \sqrt{a r}} \sum_{n=-\infty}^{\infty} Q_{n-1 / 2}(\chi) \mathrm{e}^{l n\left(\psi-\psi^{\prime}\right)},
\end{aligned}
$$

where the latter form uses [6] $Q_{n-1 / 2}(\chi)=Q_{-n-1 / 2}(\chi)$. This $V_{\delta}$ must, of course, be just the Coulomb potential $q /\left(4 \pi \varepsilon_{0}\left|\mathbf{r}-\mathbf{r}^{\prime}\right|\right)$, where in cylindrical coordinates $\mathbf{r}=$ $(r, z, \psi)$ and $\mathbf{r}^{\prime}=\left(a, 0, \psi^{\prime}\right)$. Therefore, writing $r^{\prime}$ instead of $a$ for greater symmetry, we have the mathematical identity

$\frac{1}{\left|\mathbf{r}-\mathbf{r}^{\prime}\right|}=\frac{1}{\pi \sqrt{r r^{\prime}}} \sum_{n=-\infty}^{\infty} Q_{n-1 / 2}(\chi) \mathrm{e}^{i n\left(\psi-\psi^{\prime}\right)}$

where $\chi=\left(r^{2}+r^{\prime 2}+z^{2}\right) /\left(2 r r^{\prime}\right)$. Since $\left|\mathbf{r}-\mathbf{r}^{\prime}\right|^{2}$ can be written as $r^{2}+r^{\prime 2}+z^{2}-2 r r^{\prime} \cos \left(\psi-\psi^{\prime}\right)$. Eq. (12) can be recast as

$\sum_{n=-\infty}^{\infty} Q_{n-1 / 2}(x) \mathrm{e}^{i n \phi}=\frac{\pi}{\sqrt{2(x-\cos \phi)}}$.

As pointed out by Cohl et al. [6] this result, valid for any $x \geqslant 1$ and any angle $\phi$, was proved by Heine in 1881 .

It may seem that little is gained by writing the inverse distance between two points as this apparently more complicated infinite sum, but Eq. (12) is claimed [6] to be the basis for computationally advantageous methods in astrophysics and possibly also in atomic physics. One reason for this is that when applied to a spatial distribution of charge (or mass) each term in this sum is effectively dealing with a ring and not just a point. Also the sequence of Legendre functions can be efficiently calculated because they satisfy simple recurrence relations [7].

The potential due to a uniform or sinusoidal charge distribution around a circle can also be found by direct integration. If the line charge density on the circle of radius $a$, at angle $\psi^{\prime}$, is $2 \pi \varepsilon_{0} \cos n \psi^{\prime}$, then the potential at the point $\mathbf{r}=(r, z, \psi)$ is

$V_{n}=\frac{1}{2} \int_{0}^{2 \pi} \frac{\cos n \psi^{\prime}}{\left|\mathbf{r}-\mathbf{r}^{\prime}\right|} a \mathrm{~d} \psi^{\prime}$

where $\mathbf{r}^{\prime}=\left(a, 0, \psi^{\prime}\right)$, as in the discussion leading to Eq. (12). Using $\left|\mathbf{r}-\mathbf{r}^{\prime}\right|=\sqrt{2 \operatorname{ar}\left(\chi-\cos \left(\psi-\psi^{\prime}\right)\right)}$, as before, and comparing with Eq, 9, gives the integral [10]

$\int_{0}^{\pi} \frac{\cos n \phi}{\sqrt{2(x-\cos \phi)}} \mathrm{d} \phi=Q_{n-1 / 2}(x)$.

This integral is equivalent to Heine's Eq. (13) through Fourier theory.

\subsection{Extension to charges inside a torus}

These methods can be extended to deal with charge on the reference circle inside a grounded conducting torus with constant $\eta$, say $\eta=\eta_{0}$. For a uniform distribution of charge on the reference circle, the potential is

$V=\sqrt{a / r}\left[Q_{-1 / 2}(\chi)-P_{-1 / 2}(\chi) Q_{-1 / 2}\left(\chi_{0}\right) / P_{-1 / 2}\left(\chi_{0}\right)\right]$,

where $\chi_{0}:=\operatorname{coth} \eta_{0}$. This is the correct potential because it satisfies Laplace's equation, becomes zero at $\eta=\eta_{0}$, and has the same logarithmic singularity on the reference circle as in Eq. (8) because $\sqrt{a / r} P_{-1 / 2}(\chi)$ is not singular there. The line charge density on the circle is therefore $2 \pi \varepsilon_{0}$, as before.

Similarly, for a line charge density varying as $\cos n \psi$ the potential is

$$
\begin{aligned}
V= & \sqrt{a / r}\left[Q_{n-1 / 2}(\chi)\right. \\
& \left.-P_{n-1 / 2}(\chi) Q_{n-1 / 2}\left(\chi_{0}\right) / P_{n-1 / 2}\left(\chi_{0}\right)\right] \cos n \psi .
\end{aligned}
$$


And for an arbitrary distribution of charge around the reference circle, one can find the potential as a sum over these terms using the Fourier series of the line charge density. In particular, for a point charge $q$ at angle $\psi^{\prime}$ on the reference circle, the potential is

$$
\begin{aligned}
V= & \frac{q}{4 \pi^{2} \varepsilon_{0} \sqrt{a r}} \sum_{n=0}^{\infty} \delta_{n}\left[Q_{n-1 / 2}(\chi)\right. \\
& \left.-P_{n-1 / 2}(\chi) Q_{n-1 / 2}\left(\chi_{0}\right) / P_{n-1 / 2}\left(\chi_{0}\right)\right] \cos n \psi,
\end{aligned}
$$

following the analysis leading to Eq. (11). The part involving $Q_{n-1 / 2}(\chi)$ is just the Coulomb potential due to the charge $q$ while the part involving $P_{n-1 / 2}(\chi)$ is the potential due to the induced charge on the torus.

\section{Charges between intersecting conducting planes}

Take the rotational axis of the toroidal system to lie on the intersection of the planes and let one of the planes be the origin of the azimuthal angle, $\psi=0$. If $\beta$ is the angle between the planes, then the second plane is at $\psi=\beta$. The solutions of Laplace's equation (except on the reference circle) that become zero on both planes are $\sqrt{a / r} Q_{n \pi / \beta-1 / 2}$ $(\chi) \sin (n \pi \psi / \beta)$, where $n=1,2,3, \ldots$. These correspond to a line-charge density on the reference circle of $2 \pi \varepsilon_{0} \sin$ $(n \pi \psi / \beta)$. Again one could construct the potential for an arbitrary charge along the portion of the reference circle between the planes using its Fourier series. For a point charge we need the delta-function appropriate for functions that are zero at $\psi=0$ and at $\psi=\beta$, and that is [11]

$\delta\left(\psi-\psi^{\prime}\right)=\frac{2}{\beta} \sum_{n=1}^{\infty} \sin \frac{n \pi \psi}{\beta} \sin \frac{n \pi \psi^{\prime}}{\beta}$.

For a point charge $q$ at $\psi^{\prime}$ we require a line charge density $(q / a) \delta\left(\psi-\psi^{\prime}\right)$ and therefore the potential is

$V=\frac{1}{4 \pi \varepsilon_{0}} \frac{4 q}{\beta \sqrt{a r}} \sum_{n=1}^{\infty} Q_{n \pi / \beta-1 / 2}(\chi) \sin \frac{n \pi \psi}{\beta} \sin \frac{n \pi \psi^{\prime}}{\beta}$.

This problem of a point charge between two intersecting conducting planes appears in Batygin's collection [12]. There cylindrical coordinates were used to give a completely different expression for the potential.

An interesting special case is where $\beta=2 \pi$. This is the case of a point charge outside a single semi-infinite conducting plane with a straight boundary (a half plane). The potential is

$V=\frac{1}{4 \pi \varepsilon_{0}} \frac{2 q}{\pi \sqrt{a r}} \sum_{n=1}^{\infty} Q_{1 / 2 n-1 / 2}(\chi) \sin \frac{1}{2} n \psi \sin \frac{1}{2} n \psi^{\prime}$.

Inserting $2 \sin \frac{1}{2} n \psi \sin \frac{1}{2} n \psi^{\prime}=\cos \frac{1}{2} n\left(\psi-\psi^{\prime}\right)-\cos \frac{1}{2} n\left(\psi+\psi^{\prime}\right)$ shows that we require sums of the form $\sum_{n=1}^{\infty} Q_{1 / 2 n-1 / 2}$ $(\chi) \cos \frac{1}{2} n \phi$. In the Appendix it is shown that

$S(\chi, \phi)$

$$
\begin{aligned}
& :=\sum_{n=1}^{\infty} Q_{1 / 2 n-1 / 2}(\chi) \cos \frac{1}{2} n \phi+\frac{1}{2} Q_{-1 / 2}(\chi) \\
& =\frac{1}{\sqrt{2(\chi-\cos \phi)}}\left[\frac{1}{2} \pi+\arctan \left(\frac{2 \cos \frac{1}{2} \phi}{\sqrt{2(\chi-\cos \phi)}}\right)\right] .
\end{aligned}
$$

Therefore, the potential $V(\mathbf{r})$ at position $\mathbf{r}$ with cylindrical coordinates $(r, z, \psi)$ due to a point charge $q$ at $\left(r^{\prime}, z^{\prime}, \psi^{\prime}\right)$ and a conducting half plane at $\psi=0$ is

$V(\mathbf{r})=\frac{1}{4 \pi \varepsilon_{0}} \frac{q}{\pi \sqrt{r r^{\prime}}}\left[S\left(\chi, \psi-\psi^{\prime}\right)-S\left(\chi, \psi+\psi^{\prime}\right)\right]$,

where $\chi=\left[r^{2}+r^{\prime 2}+\left(z-z^{\prime}\right)^{2}\right] /\left(2 r r^{\prime}\right)$. An expression equivalent to Eq. (22) has been found using a different method [15]. Fig. 3 shows the potential (on a plane of constant $z$ ) for an example of this system.

(a)
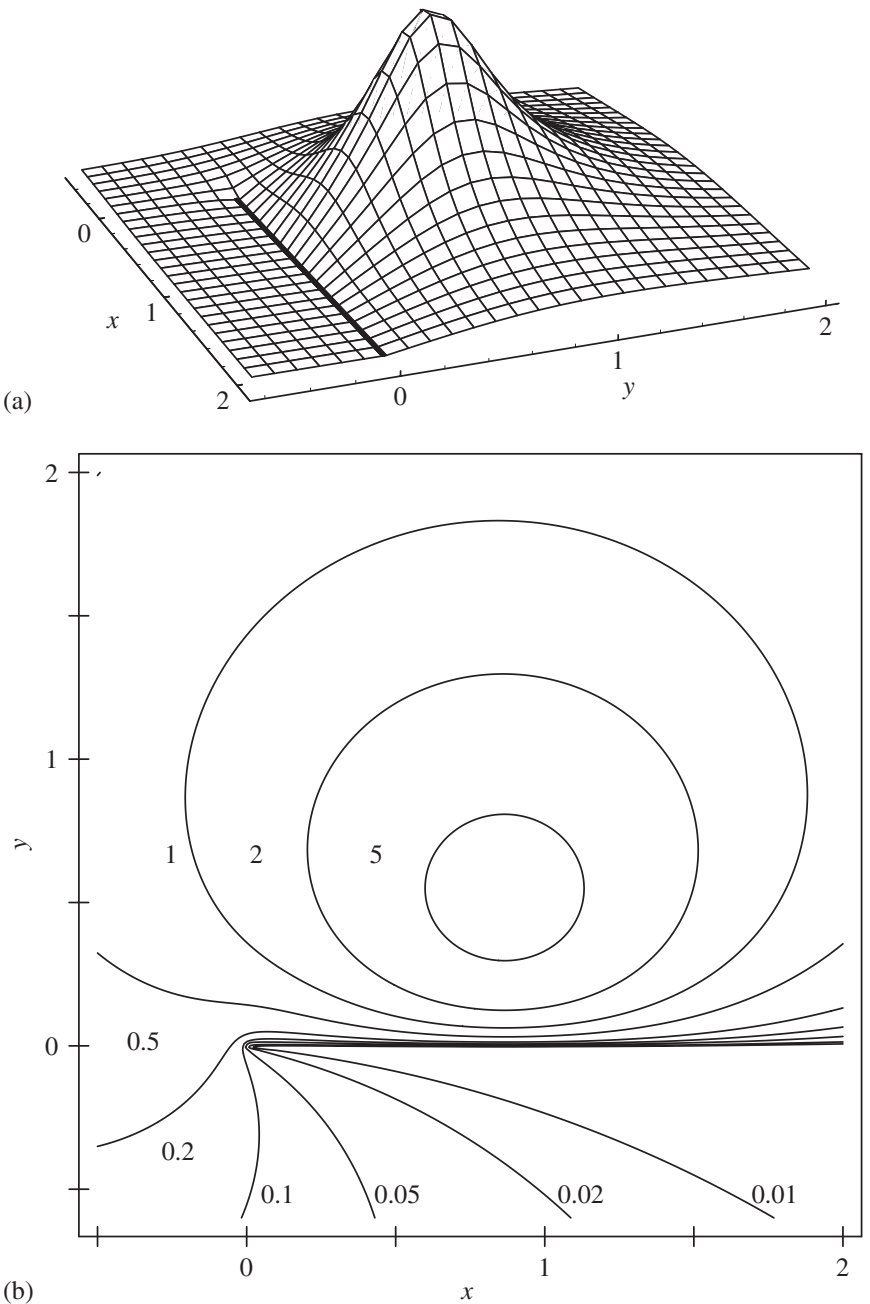

Fig. 3. The potential due to a point charge outside a conducting half plane $\mathrm{P}$. Here $\mathrm{P}$ has $y=0, x>0$ and the charge is one unit from the edge of $\mathrm{P}$ and half a unit from $\mathrm{P}$ (so that $\psi=30^{\circ}$ ). The diagrams show the potential on the plane perpendicular to the edge of $\mathrm{P}$ and 0.3 units from the charge. (a) General features of the potential. (b) Some equipotential curves; but note that the values of the potential are relatively small behind $\mathrm{P}$. [All parameters are the same in the two diagrams.] 


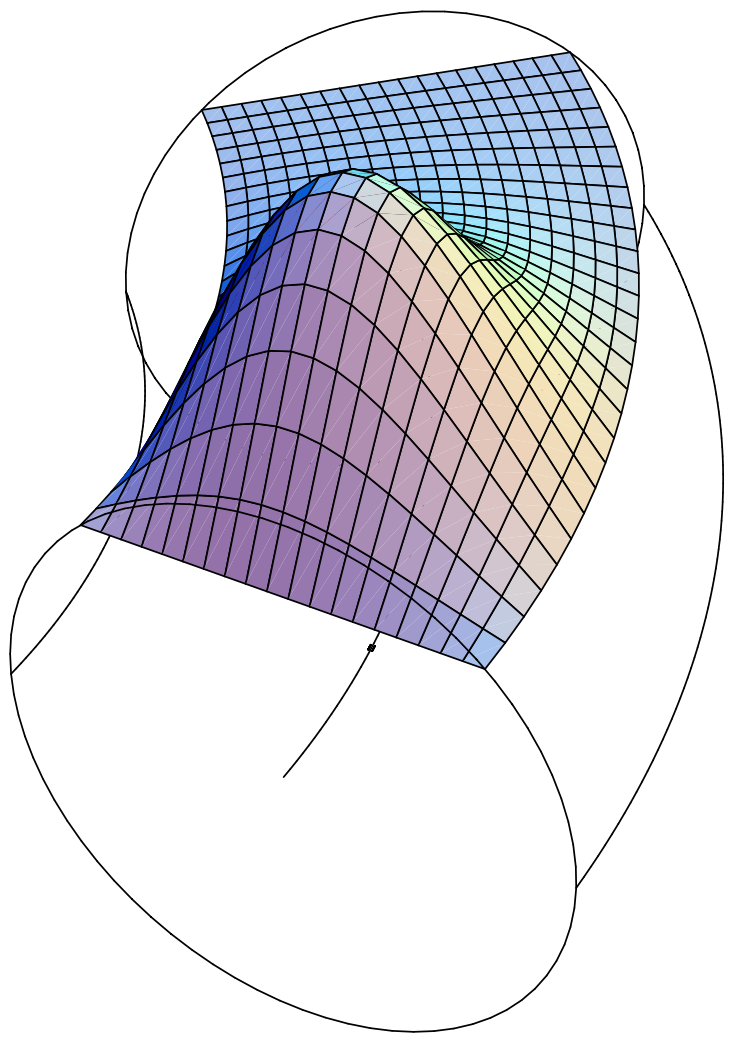

Fig. 4. The potential due to a point charge inside a portion of a conducting torus and on the reference circle of the torus. The portion is closed by conducting planar ends at $\psi=0$ and $\psi=\beta$. In this example, $\eta=2, \beta=45^{\circ}$ and the charge is at $\psi=10^{\circ}$. The potential is shown for the plane $z=0.2 a$. [For $\eta=2$ the inner radius of the torus is 0.2757...a.] Part of the reference circle is shown and the position of the charge is indicated by a heavy dot. Also shown are the two part-circles where the torus intersects the $z=0$ plane.

The methods of this section can be combined with those in Section 5 to deal with charges on the reference circle inside a portion of a torus (with $\eta=\eta_{0}$ ) closed off by conducting planar ends at $\psi=0$ and $\psi=\beta$. Thus, the potential when there is a point charge $q$ at $\psi=\psi^{\prime}$ is

$$
\begin{aligned}
V= & \frac{1}{4 \pi \varepsilon_{0}} \frac{4 q}{\beta \sqrt{r r^{\prime}}} \sum_{n=1}^{\infty}\left[Q_{n \pi / \beta-1 / 2}(\chi)-P_{n \pi / \beta-1 / 2}(\chi)\right. \\
& \left.\times Q_{n \pi / \beta-1 / 2}\left(\chi_{0}\right) / P_{n \pi / \beta-1 / 2}\left(\chi_{0}\right)\right] \sin \frac{n \pi \psi}{\beta} \sin \frac{n \pi \psi^{\prime}}{\beta} .
\end{aligned}
$$

An example is shown in Fig. 4.

\section{Reconstructing the $\mathbf{P}$-solutions from their singularities}

The solutions $V_{n}^{P}:=\sqrt{a / r} P_{n-1 / 2}(\operatorname{coth} \eta) \cos n \psi$ of Laplace's equation correspond to a charge distribution along the $z$-axis. Here, the potential will be reconstructed from that charge distribution by adding the contributions from each small part of the $z$-axis.

First, consider the case where $n=0$, so that $V_{0}^{P}:=\sqrt{a / r} P_{-1 / 2}(\operatorname{coth} \eta) . \quad$ From Table 2, $V_{0}^{P} \sim-$ $(2 a / \pi)\left(a^{2}+z^{2}\right)^{-1 / 2} \ln r$ close to the $z$-axis. This corresponds to a line charge density of $\lambda(z)=4 a \varepsilon_{0}\left(a^{2}+z^{2}\right)^{-1 / 2}$ on the $z$-axis. This is not integrable to a finite amount of charge. Integrating the Coulomb potential from each small segment of the $z$-axis gives

$$
\begin{aligned}
V_{0}^{P} & =\frac{1}{4 \pi \varepsilon_{0}} \int_{-\infty}^{\infty} \frac{\lambda\left(z^{\prime}\right) \mathrm{d} z^{\prime}}{\sqrt{r^{2}+\left(z^{\prime}-z\right)^{2}}} \\
& =\frac{a}{\pi} \int_{-\infty}^{\infty} \frac{\mathrm{d} z^{\prime}}{\sqrt{a^{2}+z^{\prime 2}} \sqrt{r^{2}+\left(z^{\prime}-z\right)^{2}}} .
\end{aligned}
$$

The correctness of the relation

$$
\begin{aligned}
\frac{a}{\pi} \int_{-\infty}^{\infty} \frac{\mathrm{d} z^{\prime}}{\sqrt{a^{2}+z^{\prime 2}} \sqrt{r^{2}+\left(z^{\prime}-z\right)^{2}}} \\
=\sqrt{\frac{a}{r}} P_{-1 / 2}\left(\frac{a^{2}+r^{2}+z^{2}}{2 a r}\right)
\end{aligned}
$$

confirms that the solution $V_{0}^{P}$ is just the potential due to the charge density $\lambda(z)$ along the $z$-axis. I have not found the integral in Eq. (24) in any of the standard collections, and Mathematica and Maple fail on it although in principle it can be treated as an elliptic integral. It is a special case $(n=0)$ of Eq. (27) proved below. The relation between $P_{-1 / 2}$ and the complete elliptic integral [7] is well known, and hence

$$
\begin{aligned}
& \int_{-\infty}^{\infty} \frac{\mathrm{d} z^{\prime}}{\sqrt{a^{2}+z^{\prime 2}} \sqrt{r^{2}+\left(z^{\prime}-z\right)^{2}}} \\
& =\frac{4}{\sqrt{(r+a)^{2}+z^{2}}} K\left(\frac{(r-a)^{2}+z^{2}}{(r+a)^{2}+z^{2}}\right) .
\end{aligned}
$$

For $n=1,2,3, \ldots$ Table 2 shows that, for $r \ll a$,

$V_{n}^{P}:=\sqrt{a / r} P_{n-1 / 2}(\operatorname{coth} \eta) \cos n \psi \sim M(z) r^{-n} \cos n \psi$,

where $\quad M(z):=C_{n} a^{-n+1}\left(z^{2}+a^{2}\right)^{n-1 / 2}$. The potential $r^{-n} \cos n \psi$ corresponds to what one might call a cylindrical $n$-pole. It satisfies Laplace's equation except at $r=0$ and corresponds to $2 n$ lines of charge, all parallel to the $z$-axis, alternatively positive and negative, arranged to make a cylinder coaxial with the $z$-axis, in the limit where the radius of the cylinder tends to zero. So Eq. (26) implies that $V_{n}^{P}$ is produced by an $n$-pole of strength $M(z)$. The radial component $E_{r}$ of the electric field due to the potential $r^{-n} \cos n \psi$ is $E_{r}=n r^{-n-1} \cos n \psi$. To generate $V_{n}^{P}$ consider a cylinder of radius $b \ll a$, with surface charge density $\sigma(z, \psi)=\varepsilon_{0} E_{r} M(z)=\varepsilon_{0} n M(z) b^{-n-1} \cos n \psi$. To calculate the potential due to this cylinder of charge, take a slice of height $\mathrm{d} z^{\prime}$ at $z=z^{\prime}$. It will be a circle of radius $b$ with line charge density $2 \sigma\left(z^{\prime}, \psi\right) \mathrm{d} z^{\prime}$. (The extra factor of 2 is required because only half of the charge on the cylinder contributes to the outward field.) We already know [Eq. (9)] that the potential at $(r, z, \psi)$ due to a circle of radius $b$ at $z^{\prime}$ with line charge density $2 \pi \varepsilon_{0} \cos n \psi$ is $\sqrt{b / r} Q_{n-1 / 2}\left(r^{2}+\left(z-z^{\prime}\right)^{2}+b^{2}\right) /(2 b r) \cos n \psi$, and from Table 2 , for $R \gg b$ this potential will become $D_{n}\left(b^{2} / R^{2}\right)^{n+1 / 2}(r / b)^{n}$ where $R^{2}=r^{2}+\left(z-z^{\prime}\right)^{2}$. 
Therefore, combining the contributions from all these slices, and using $C_{n} D_{n}=1 / n$,

$\frac{r^{n}}{\pi a^{n-1}} \int_{-\infty}^{\infty} \frac{\left(a^{2}+z^{\prime 2}\right)^{n-1 / 2} \mathrm{~d} z^{\prime}}{\left(r^{2}+\left(z-z^{\prime}\right)^{2}\right)^{n+1 / 2}}=\sqrt{\frac{a}{r}} P_{n-1 / 2}\left(\frac{a^{2}+r^{2}+z^{2}}{2 a r}\right)$.

The correctness of this relation shows that $V_{n}^{P}$ is solely due to the $n$-pole distribution along the $z$-axis.

To verify Eq. (27) put $a=1$ for simplicity and substitute $u=\left(\alpha z^{\prime}+1\right) /\left(z^{\prime}-\alpha\right) \quad$ into $\quad I:=\int_{-\infty}^{\infty}\left(z^{\prime 2}+1\right)^{v}\left[\left(z^{\prime}-z\right)^{2}+\right.$ $\left.r^{2}\right]^{-v-1} \mathrm{~d} z^{\prime}$, with $\alpha-\alpha^{-1}=z^{-1}\left(r^{2}+z^{2}-1\right)$, to give $I=2(1-z / \alpha)^{-v-1} \int_{0}^{\infty}\left(u^{2}+1\right)^{v}\left(u^{2}+t^{2}\right)^{-v-1} \mathrm{~d} u$, where $t^{2}=$ $(1+\alpha z) /(1-z / \alpha)$. But $(1+\alpha z)(1-z / \alpha)=r^{2}$ so $t=(1+\alpha z) / r$ and $t^{-1}=(1-z / \alpha) / r$. Substituting $u=x^{2}$ in $I$ puts it into a standard hypergeometric form [13] giving $I=$ $\pi(t r)^{-v-1} F\left(v+1, \frac{1}{2} ; 1 ; 1-t^{-2}\right)$, which can be expressed as [14] $I=\pi r^{-v-1} P_{v}\left(\frac{1}{2}\left[t+t^{-1}\right]\right)$, and $t+t^{-1}=\left(r^{2}+z^{2}+1\right) / r$. Now restoring $a$ gives Eq. (27).

It is remarkable that the integral in Eq. (27) can be so simply expressed in terms of the Legendre function, and we have shown that the relation is valid for any $n$, even though the context here requires $n$ to be an integer for continuity in $\psi$.

\section{Conclusion}

An alternative method of separating variables in toroidal coordinates provides a simple route to a basis of solutions of Laplace's equation appropriate for boundary conditions that are independent of the spherical coordinate $\theta$. This gives the potential for a class of problems in electrostatics, and reconstructing the potential from the charge distributions (corresponding to singularities in the solutions) gives rise to some relations involving Legendre functions.

\section{Appendix A. Sum of series over Legendre- $Q$ functions}

We require sums of the form

$$
\begin{aligned}
& \sum_{n=1}^{\infty} Q_{1 / 2 n-1 / 2}(\chi) \cos \frac{1}{2} n \phi \\
& =\sum_{n=0}^{\infty} Q_{n}(\chi) \cos \left(n+\frac{1}{2}\right) \phi+\sum_{n=1}^{\infty} Q_{n-1 / 2}(\chi) \cos n \phi .
\end{aligned}
$$

The second of these sums can be found from Heine's Eq. (13)

$$
\sum_{n=1}^{\infty} Q_{n-1 / 2}(\chi) \cos n \phi+\frac{1}{2} Q_{-1 / 2}(\chi)=\frac{\frac{1}{2} \pi}{\sqrt{2(\chi-\cos \phi)}} .
$$

From the generating relation (see a few lines below) we can deduce the first

$$
\begin{aligned}
& \sum_{n=0}^{\infty} Q_{n}(\chi) \cos \left(n+\frac{1}{2}\right) \phi \\
& \quad=\frac{1}{\sqrt{2(\chi-\cos \phi)}} \arctan \left(\frac{2 \cos \frac{1}{2} \phi}{\sqrt{2(\chi-\cos \phi)}}\right) .
\end{aligned}
$$

Adding these gives

$$
\begin{aligned}
& S(\chi, \phi) \\
& \quad:=\sum_{n=1}^{\infty} Q_{1 / 2 n-1 / 2}(\chi) \cos \frac{1}{2} n \phi+\frac{1}{2} Q_{-1 / 2}(\chi) \\
& \quad=\frac{1}{\sqrt{2(\chi-\cos \phi)}}\left[\frac{1}{2} \pi+\arctan \left(\frac{2 \cos \frac{1}{2} \phi}{\sqrt{2(\chi-\cos \phi)}}\right)\right] .
\end{aligned}
$$

\section{A.1. Derivation of Eq. (A.3)}

The generating relation $[16,17]$ for $Q_{n}(w)$ is

$$
\begin{aligned}
& \sum_{n=0}^{\infty} h^{n} Q_{n}(w) \\
& \quad=\frac{1}{\sqrt{1-2 w h+h^{2}}} \ln \left(\frac{w-h+\sqrt{1-2 w h+h^{2}}}{\sqrt{w^{2}-1}}\right) .
\end{aligned}
$$

If $h=\mathrm{e}^{i \phi}$, then $1-2 w h+h^{2}=-2 \mathrm{e}^{i \phi}(w-\cos \phi)$. We require $w>1$ and therefore, with $u:=\sqrt{2(w-\cos \phi)}$,

$$
\begin{aligned}
\sum_{n=0}^{\infty} & Q_{n}(w) \mathrm{e}^{\imath(n+1 / 2) \phi} \\
& =\frac{l}{u} \ln \left(\frac{w-\mathrm{e}^{\imath \phi}+\imath \mathrm{e}^{1 / 2 l \phi} u}{\sqrt{w^{2}-1}}\right) \\
& =\frac{l}{u} \ln \left(\frac{u-2 \sin \frac{1}{2} \phi}{2 \sqrt{w^{2}-1}}\left[u+2 \imath \cos \frac{1}{2} \phi\right]\right),
\end{aligned}
$$

and the real and imaginary parts of this give

$$
\begin{aligned}
& \sum_{n=0}^{\infty} Q_{n}(w) \cos \left(n+\frac{1}{2}\right) \phi=\frac{1}{u} \arctan \left(\frac{2 \cos \frac{1}{2} \phi}{u}\right) \\
& \sum_{n=0}^{\infty} Q_{n}(w) \sin \left(n+\frac{1}{2}\right) \phi \\
& =\frac{-1}{u} \ln \left(\frac{u-2 \sin \frac{1}{2} \phi}{\sqrt{2(w-1)}}\right)=\frac{1}{u} \operatorname{arcsinh}\left(\frac{2 \sin \frac{1}{2} \phi}{\sqrt{2(w-1)}}\right) .
\end{aligned}
$$

\section{Appendix B. Comparison with the traditional separation}

The method of separation usually found in textbooks [1,4] inserts $V=\sqrt{\cosh \eta-\cos \theta} U$ into Laplace's (6) instead of $V=\sqrt{1 / r} U$ as in Section 3. This is not essentially different, because $\cosh \eta-\cos \theta=(a / r) \sin \theta$, but the separation is slightly different and leads to associated Legendre functions of $\cosh \eta$ (instead of $\operatorname{coth} \eta$ ). The result is solutions of Laplace's equation that are products of $\sqrt{\cosh \eta-\cos \theta}, \quad P_{v-1 / 2}^{\mu}(\cosh \eta)$ or $Q_{v-1 / 2}^{\mu}(\cosh \eta), \sin v \theta$ or $\cos v \theta$, and $\sin \mu \psi$ or $\cos \mu \psi$. Note that now the lower index is associated with the $\theta$ dependence (while in Section 3 it was the upper index). This means that 
in this traditional approach the simpler Legendre functions will suffice for axially symmetric situations. (Another way to see the equivalence of the two approaches to separation of the variables is to note [18] that both $\sqrt{\sinh \eta} P_{v-1 / 2}^{\mu}(\cosh \eta)$ and $\sqrt{\sinh \eta} Q_{v-1 / 2}^{\mu}(\cosh \eta)$ can be expressed as linear combinations of $P_{\mu-1 / 2}^{v}(\operatorname{coth} \eta)$ and $Q_{\mu-1 / 2}^{v}(\operatorname{coth} \eta)$. Therefore, a term (in the traditional expansion) of the form $\sqrt{\Delta} P_{v-1 / 2}^{\mu}(\cosh \eta) \sin v \theta \sin \mu \psi$ can be written, using Eq. (2), as a linear combination of two terms of the form $r^{-1 / 2} P_{\mu-1 / 2}^{v}(\operatorname{coth} \eta) \sin v \theta \sin \mu \psi$ and $r^{-1 / 2} Q_{\mu-1 / 2}^{v}(\operatorname{coth} \eta)$ $\sin v \theta \sin \mu \psi$.)

Thus for axially symmetric systems, the potential can be written as a sum of terms that are products of $\sqrt{\cosh \eta-\cos \theta}, \quad P_{n-1 / 2}(\cosh \eta)$ or $Q_{n-1 / 2}(\cosh \eta)$, and $\sin n \theta$ or $\cos n \theta$. (The continuity in $\theta$ requires that $v=0,1,2, \ldots$.)

\section{B.1. Example: The potential outside a charged conducting torus}

The $Q_{n-1 / 2}(\cosh \eta)$ are too divergent at $\eta=0$ (which corresponds to the $z$-axis). The boundary condition that $V=V_{0}$ (a constant) for $\eta=\eta_{0}$ (specifying the conducting toroidal surface), being even in $\theta$, excludes terms in $\sin \theta$. Thus, the potential outside the torus must have the form

$V=\sqrt{\cosh \eta-\cos \theta} \sum_{n=0}^{\infty} a_{n} P_{n-1 / 2}(\cosh \eta) \cos n \theta$.

Imposing the condition that $V=V_{0}$ for $\eta=\eta_{0}$ is easily done by comparing this equation with Heine's Eq. (13) in the form

$\pi=\sqrt{2(x-\cos \theta)} \sum_{n=0}^{\infty} \delta_{n} Q_{n-1 / 2}(x) \cos n \theta$,

where $\delta_{0}=1$ and $\delta_{n}=2$ for $n>0$. Thus, the potential outside the conducting torus $\eta=\eta_{0}$ at potential $V=V_{0}$ is

$$
\begin{aligned}
V= & \frac{V_{0}}{\pi} \sqrt{2(\cosh \eta-\cos \theta)} \sum_{n=0}^{\infty} \delta_{n} \\
& \times \frac{Q_{n-1 / 2}\left(\cosh \eta_{0}\right)}{P_{n-1 / 2}\left(\cosh \eta_{0}\right)} P_{n-1 / 2}(\cosh \eta) \cos n \theta .
\end{aligned}
$$

\section{B.2. Further examples}

These deal with some cases where there are charges inside a torus.

1. The potential inside the torus $\eta=\eta_{0}$, when there is a uniformly charged ring on the reference circle $(r=a$, $z=0$ ), has the form

$$
\begin{aligned}
V= & \sqrt{\cosh \eta-\cos \theta}\left[P_{-1 / 2}(\cosh \eta)\right. \\
& \left.-Q_{-1 / 2}(\cosh \eta) P_{-1 / 2}\left(\cosh \eta_{0}\right) / Q_{-1 / 2}\left(\cosh \eta_{0}\right)\right] .
\end{aligned}
$$

The line-charge density can be deduced from the logarithmic singularity in $P_{-1 / 2}(\cosh \eta)$ at the ring, as for Eq. (8). This case was also considered in Section 5; it can be treated by either approach because the boundary conditions do not depend on $\theta$ or $\psi$. The two different looking results, Eq. (B.4) and Eq. (15), are equivalent because [19]

$P_{-1 / 2}(\cosh \eta)=\frac{1}{\pi} \sqrt{\frac{2}{\sinh \eta}} Q_{-1 / 2}(\operatorname{coth} \eta)$

$Q_{-1 / 2}(\cosh \eta)=\pi \sqrt{\frac{2}{\sinh \eta}} P_{-1 / 2}(\operatorname{coth} \eta)$.

2. Similarly,

$$
\begin{aligned}
V= & \sqrt{\cosh \eta-\cos \theta}\left[P_{1 / 2}(\cosh \eta)\right. \\
& \left.-Q_{1 / 2}(\cosh \eta) P_{1 / 2}\left(\cosh \eta_{0}\right) / Q_{1 / 2}\left(\cosh \eta_{0}\right)\right] \cos \theta
\end{aligned}
$$

corresponds to a uniform line dipole around the reference circle inside the torus. The orientation of the dipole can be arbitrarily changed since the latter $\cos \theta$ can be replaced by $\cos \left(\theta-\theta_{0}\right)$.

3. The potential between two tori (with the same reference circle $r=a, z=0$ ) held at different potentials can be expressed as a sum of the form

$$
\begin{aligned}
V= & \sqrt{\cosh \eta-\cos \theta} \sum_{n=0}^{\infty}\left[a_{n} P_{n-1 / 2}(\cosh \eta)\right. \\
& \left.+b_{n} Q_{n-1 / 2}(\cosh \eta)\right] \cos n \theta
\end{aligned}
$$

and the coefficients $a_{n}$ and $b_{n}$ can be found by solving the two linear equations that come from $V=V_{0}$ at $\eta=\eta_{0}$ and $V=V_{1}$ at $\eta=\eta_{1}$, and comparing with Eq. (B.2) in each case.

\section{References}

[1] P. Moon, D.E. Spencer, Field Theory Handbook, Springer, Berlin, 1961.

[2] P. Moon, D.E. Spencer, Field Theory Handbook, Springer, Berlin, pp. 112-115.

[3] J. Vanderlinde, Classical Electromagnetic Theory, Wiley, New York, 1993, pp. 356-360.

[4] W.R. Smythe, Static and Dynamic Electricity, McGraw-Hill, London, 1939, p. 60.

[5] J.A. Stratton, Electromagnetic Theory, McGraw-Hill, London, 1941, p. 218.

[6] H.S. Cohl, J.E. Tohline, A.R.P. Rau, H.M. Srivastava, Astron. Nachr. 321 (5/6) (2000) 363-372.

[7] M. Abramowitz, I.A. Stegun, Handbook of Mathematical Functions, National Bureau of Standards, 1964 (Chapter 8).

[8] The asymptotic behavior of the Legendre functions can be gleaned from Ref. [7], but an easier source to use is at the web address: $<$ functions.wolfram.com/HypergeometricFunctions $>$.

[9] G. Barton, Elements of Greens Functions and Propagation, Oxford University Press, Oxford, 1989 (Eq. 1.3.11).

[10] N.N. Lebedev, Special Functions and their Applications, PrenticeHall, Englewood Cliffs, NJ, 1965, p. 188.

[11] G. Barton, Elements of Greens Functions and Propagation, Oxford University Press, Oxford, 1989 (Eq. 1.3.9).

[12] V.V. Batygin, I.N. Toptygin, Problems in Electrodynamics, Academic Press, New York, 1962, pp. 45-46.

[13] A. Erdlyi (Ed.), Higher Transcendental Functions, vol. I, McGrawHill, London, 1953, p. 115, Eq. (5). 
[14] A. Erdlyi (Ed.), Higher Transcendental Functions, vol. I, McGrawHill, London, 1953, p. 173, Eq. (5).

[15] K.I. Nikoskinen, I.V. Lindell, IEEE Trans. Antennas Propagation 43 (2) (1995) 179-187.

[16] E.T. Whittaker, G.N. Watson, A Course of Modern Analysis, fourth ed., Cambridge University Press, Cambridge, 1962, p. 321.
[17] E.W. Hobson, The Theory of Spherical and Ellipsoidal Harmonics, Cambridge University Press, Cambridge, 1939, p. 69.

[18] H.S. Cohl, J.E. Tohline, A.R.P. Rau, H.M. Srivastava, Astron. Nachr. 321 (5/6) (2000) 363-372 (Eq. 31, 32).

[19] H.S. Cohl, J.E. Tohline, A.R.P. Rau, H.M. Srivastava, Astron. Nachr. 321 (5/6) (2000) 363-372 (Eq. 33, 34). 